\title{
Surgical Pathway Seeding of Clivo-Cervical Chordomas
}

\author{
Alfred Marc Calo lloreta ${ }^{1}$ Gurston G. Nyquist ${ }^{1} \quad$ Mark Friedel $^{1} \quad$ Christopher Farrell $^{2}$ Marc R. Rosen ${ }^{1}$ \\ James J. Evans ${ }^{2}$ \\ ${ }^{1}$ Department of Otolaryngology-Head and Neck Surgery, Thomas \\ Jefferson University Hospital, Philadelphia, Pennsylvania, \\ United States \\ ${ }^{2}$ Division of Neuro-Oncologic Neurosurgery, Department of \\ Neurological Surgery, Thomas Jefferson University Hospital, \\ Philadelphia, Pennsylvania, United States \\ Address for correspondence Alfred Marc Calo Iloreta, MD, \\ Department of Otolaryngology- Head and Neck Surgery, Thomas \\ Jefferson University Hospital, 925 Chestnut St., 6th Floor, Philadelphia, \\ PA 19107, United States (e-mail: jaymarc.iloreta@gmail.com). \\ J Neurol Surg Rep 2014;75:e246-e250.
}

\begin{abstract}
Keywords

- skull base

- surgical seeding

- endoscopic surgery

- chordoma

- clivus

Objective Clival chordomas are slow-growing aggressive tumors that originate from the extra-axial remnants of the notochord. Current management of these tumors use surgical resection combined with radiation therapy. Given the location and invasive nature of these tumors, complete resection is difficult. A variety of both open and endoscopic therapeutic approaches have evolved and combined with the improvements in proton therapy, long-term control of these tumors appears to be improving. However, in recent literature the relatively rare complication of surgical seeding or surgical pathway recurrence has been reported. We report a case of surgical seeding following primary resection and review the world literature regarding surgical pathway recurrence.

Study Design Retrospective chart review and review of current literature.

Methods We report a case of a patient with a large chordoma that required treatment with a staged endoscopic endonasal and external transcervical approach. The patient subsequently developed recurrent disease along the cervical skin incision due to surgical seeding. Literature review and case reports were identified by a comprehensive search of Medline for the years 1950 to 2012.

Results The overall surgical pathway recurrence rate for clival chordoma resection based on analysis of the open nonendoscopic published case studies was 14 of 497 (2.8\%).

Conclusion Tumor seeding can occur anywhere along the operative route and is often outside the field of radiotherapy. Increased awareness of this rare occurrence is necessary. The use of novel techniques to minimize exposure to tumor including primary endoscopic resection and so-called clean oncologic technique may help limit tumor seeding. Level of evidence: 4.
\end{abstract}

\section{Introduction}

Clivo-cervical chordomas are locally destructive, invasive, slow-growing tumors that originate from the extra-axial remnants of the notochord. Definitive local resection can be curative; however, these tumors are characterized by direct extension, local recurrence, and more rarely systemic and cerebrospinal fluid metastasis. Evidence has shown that received

December 8, 2013

accepted after revision

June 7, 2014

published online

November 12, 2014
DOI http://dx.doi.org/

10.1055/s-0034-1387184. ISSN 2193-6358. (c) 2014 Georg Thieme Verlag KG
Stuttgart · New York

License terms

$\circledast(1) \Theta \circledast$ 
adjuvant therapy with high-dose radiotherapy can promote local control. Failure can occur in the form of local recurrence, metastases to cerebrospinal fluid, distant sites, and regional lymph nodes. Even more increasingly rare is tumor seeding along the surgical access pathway. These sites, such as the nasal cavity and surrounding soft tissue, are often outside the treatment field of radiation and can be subject to iatrogenic tumor implantation.

We report a case of a patient with a recurrent clivo-cervical chordoma as a result of surgical pathway seeding and perform a literature review and meta-analysis of all reported cases of surgical pathway seeding of clival chordomas.

\section{Case Report}

A 47 year-old man presented with upper back pain and was found to have a very large prevertebral/retropharyngeal mass extending from the clivus to $\mathrm{C} 5$ with erosion of the second cervical vertebrae, displacement of the right vertebral artery, and partial encasement of the left vertebral artery consistent with a chordoma on biopsy in June 2004 (- Fig. 1).

The patient underwent a two-stage resection including a left anterior cervical approach for tumor resection and a planned tracheostomy for airway protection. A second-stage operation including occipitocervical fusion with a posterior approach and resection of a small amount of known residual tumor at the left aspect of $\mathrm{C} 2-\mathrm{C} 3$ neuroforamen was subsequently performed in August 2004 (-Fig. 2).

In April 2006, the patient underwent endoscopic transo$\mathrm{ral} /$ transnasal odontoidectomy and resection of recurrent chordoma. Subsequently, the patient completed protonbeam radiotherapy between March 12, 2007, and May 2, 2007, with a total dose of 76.0 cobalt Gy equivalents delivered in 38 fractions. He was then closely monitored with surveil-

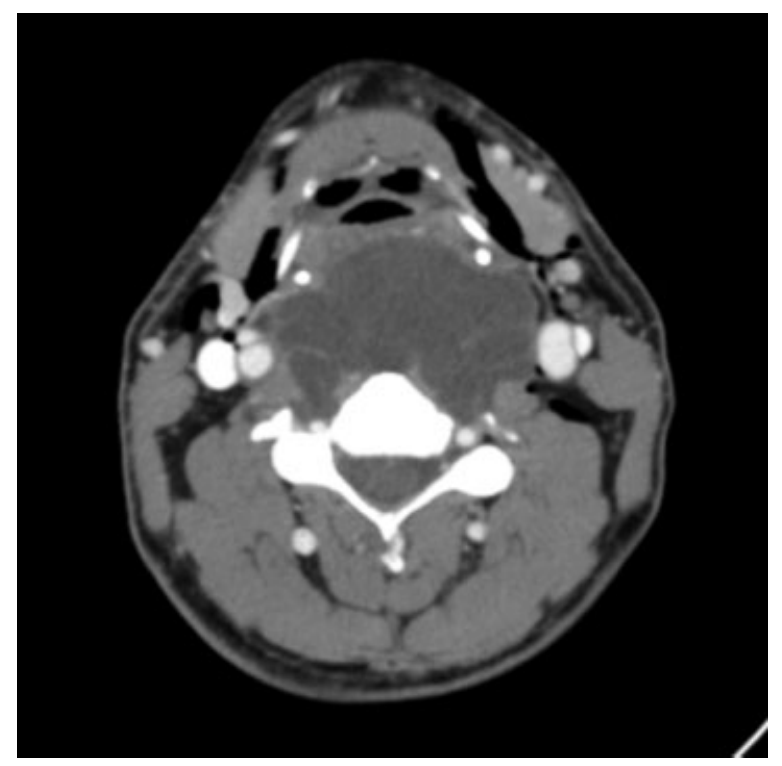

Fig. 1 Computed tomography of the neck with intravenous contrast, axial cut. Demonstration of a large retropharyngeal mass with compression of the trachea.

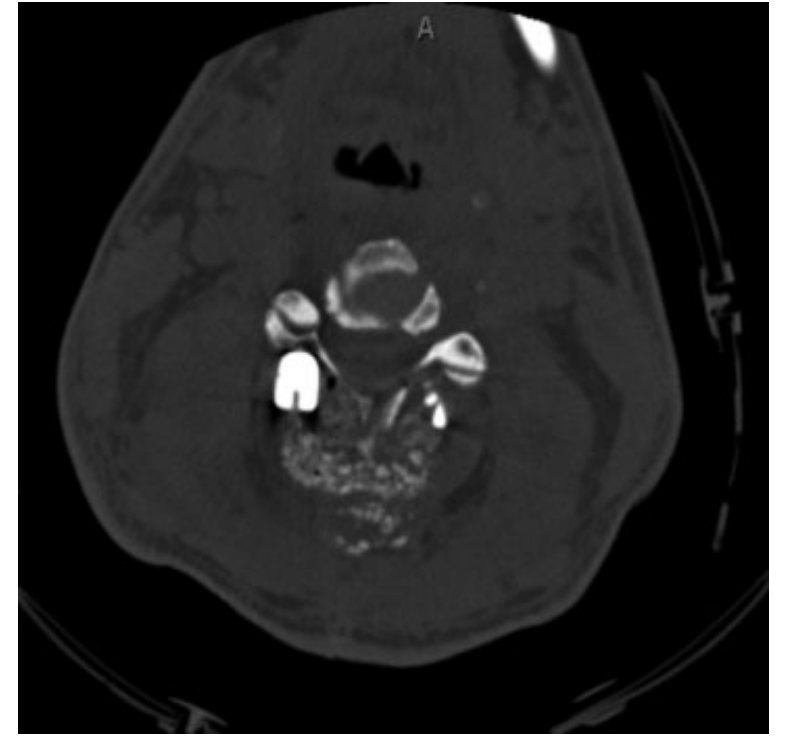

Fig. 2 Computed tomography of the cervical spine, axial cut. Demonstration of cervical spine resection bed postoperatively as well as dorsal spinal instrumented arthrodesis.

lance magnetic resonance imaging (MRI) at 6-month intervals.

In July 2010, the patient noted an area of nodularity subjacent to the anterior left neck incision site just anterior to the sternocleidomastoid muscle. MRI of the neck and cervical spine revealed a new nodularity anterior to the sternocleidomastoid muscle ( - Fig. 3). A fine-needle aspiration was performed and revealed cytologic evidence of chordoma.

The patient subsequently underwent a wide-local excision and left selective neck dissection. Final pathology revealed recurrent chordoma in the excised neck mass but no evidence of tumor within biopsy specimen of the nasopharyngeal soft tissues performed at the same time. All margins were negative at the time of resection. The patient continues to remain neurologically stable at this time without evidence of disease on the latest imaging.

\section{Methods}

A comprehensive review of the literature on surgical seeding of clival chordoma was conducted using Medline and databases with the key words clival chordoma, surgical pathway recurrence, and surgical tumor seeding. English-language studies containing case reports and series of patients were included.

\section{Results}

- Table 1 demonstrates findings from a comprehensive literature search and compares clinical data in all reported series of surgical seeding. The overall pathway recurrence rate for clival chordoma resection based on analysis of the open nonendoscopic published case studies was 14 of 497 (2.8\%). Isolated case reports were not included to determine this rate. 

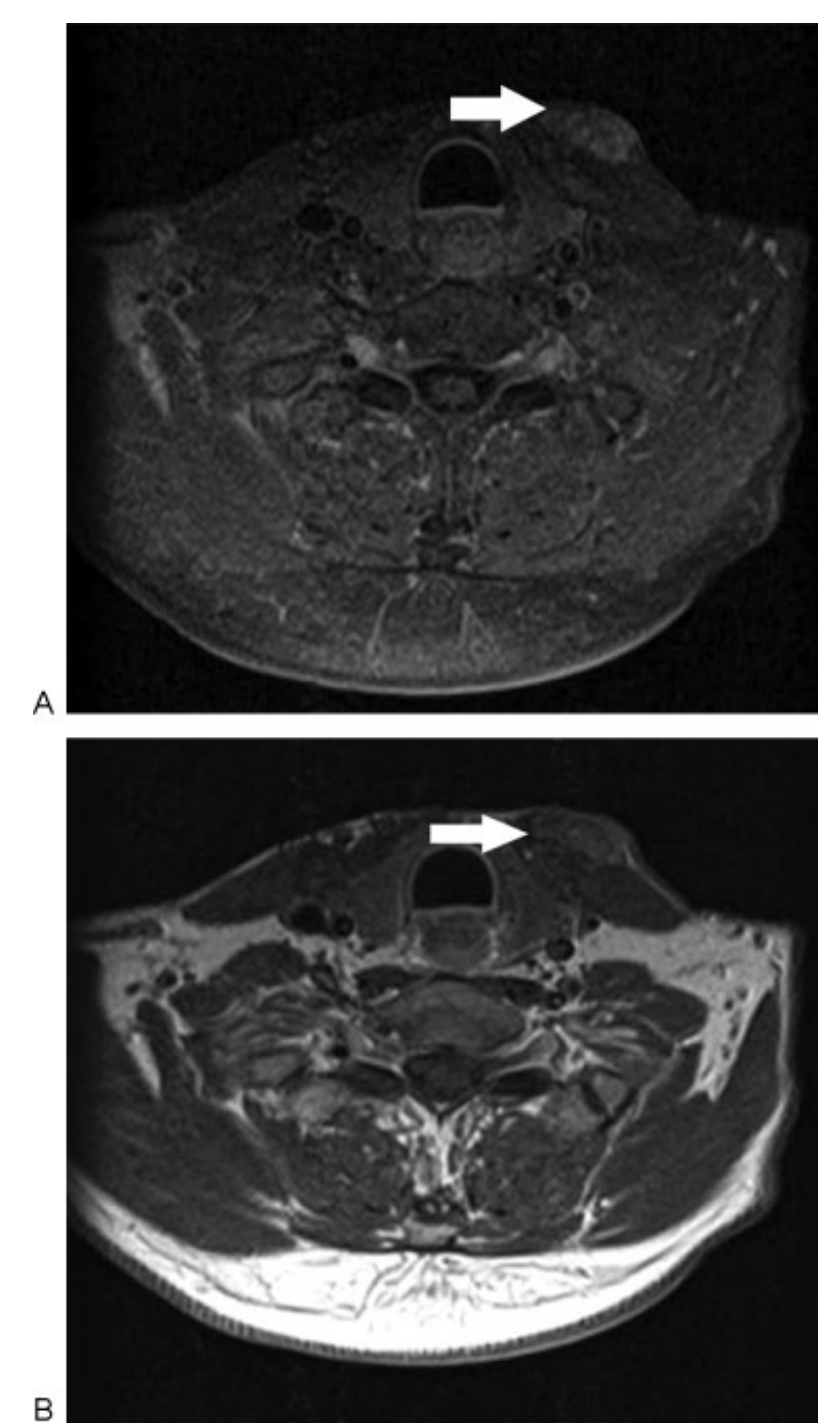

Fig. 3 (A, B) Magnetic resonance imaging of the neck, axial cut. Arrows demonstrate soft tissue recurrence of chordoma at cervical incision site.

\section{Discussion}

Clival chordomas can be very challenging cases even for the most experienced skull base teams. These tumors have a tendency to expand and spread into the surrounding tissue including critical neurovascular structures. The ability to resect these tumors and also deliver radiotherapy effectively and safely is limited by the visual pathways, carotid and basilar circulation, brainstem, and the cavernous sinus.

In general $^{1-4}$ these neoplasms behave aggressively and are associated with a high local recurrence rate, up to 21 to $29 \%$, as well as the potential to metastasize. ${ }^{1,2,5}$ Factors that can be associated with local recurrence include low postoperative radiation doses and large tumor bulk. ${ }^{1}$ The most common site of distant metastases are the lungs (58\%), lymph nodes (33\%), liver (22\%), bone (17\%), and skeletal muscle (9\%). ${ }^{6}$ Surgical pathway recurrence has a reported incidence between $1.5 \%$ and $7.3 \%$. Tumor seeding of clival chordomas has been described seven times in the literature to date. Recurrence has been documented in a variety of tissue beds (including skin, neck, nasal cavity, palate, sinuses, etc.). All of these seeding occurrences have been reported in patients who underwent open or nonendoscopic transnasal/transoral resections. From the available case series and case reports, we calculated the surgical pathway recurrence rate of $3.6 \%$ for open approaches. Excluded in this review of the literature were series that did not explicitly report on the incidence and details of surgical seeding because this complication may have been omitted. We believe that a $3.6 \%$ seeding rate reflects an accurate incidence of this complication. In a large study of 71 chordoma patients, Sen et al reported a similar incidence of 5.6\%. ${ }^{7}$

Tumor seeding is a well-described phenomenon that occurs when the spillage of tumor cell clusters implant and grow in adjacent tissue to the original tumor. Such contamination may occur at different times: preoperative rupture (spontaneous or traumatic), biopsy (needle or open), or during surgery from the tumor (rupture of the capsule or removal of the tumor in piecemeal). ${ }^{4,8,9}$ It has been described in association with breast, thoracic, gastrointestinal, thyroid, hepatic, head/neck, and urologic cancer. Dissemination and concomitant implantation of neoplastic cells following the manipulation of neoplastic lesions can occur along the open surgical bed or spread hematogenously to distant organs. Although so-called tumor spillage is an extremely common event, it rarely translates into tumor recurrence. This depends on the function of host immunity, number of cells, and the characteristics of the tumor cell and local tissue. Cytologic analysis of venous samples before, during, and after procedures show that tumor cells are commonly released into the systemic circulation. ${ }^{8,10}$ Several studies have documented up to $100 \%$ rate of circulating tumor cells following surgical manipulation; however, few of these patients develop distant metastases. ${ }^{8,10}$ The current understanding of oncologic principles dictate that a tumor consists of clones of cells with a great degree of variation in functional properties such as cell surface receptors, immunogenicity, tumorigenicity, chemosensitivity, the ability to invade local structures, and the ability to metastasize. Moreover, tumor burden does not correlate with recurrence from seeding. For a tumor cell to produce a metastasis, the cell must overcome a series of steps following its detachment from the initial tumor. It needs the ability to invade through the extracellular matrix, integrate with vascular channels and adhere to another tissue bed, avoid being destroyed by the immune system, and induce growth by encouraging neovascularization.

In the context of chordoma seeding, tumor recurrence occurs as a result of direct implantation of the tumor into adjacent tissue during the transit of instruments and tissue during the surgery. ${ }^{3}$ As demonstrated in Table 1, each of the recurrences occurred in a local tissue bed.

In an effort to prevent surgical seeding of tumor, different techniques have been described. One group fortifies the operative tunnel after the approach is completed by covering the walls with fibrin glue and large cotton patties. ${ }^{4}$ In addition, it is postulated that low-dose radiation or including the surgical ports into the radiation field may prevent surgical seeding from occurring. Our patient did not receive radiation 

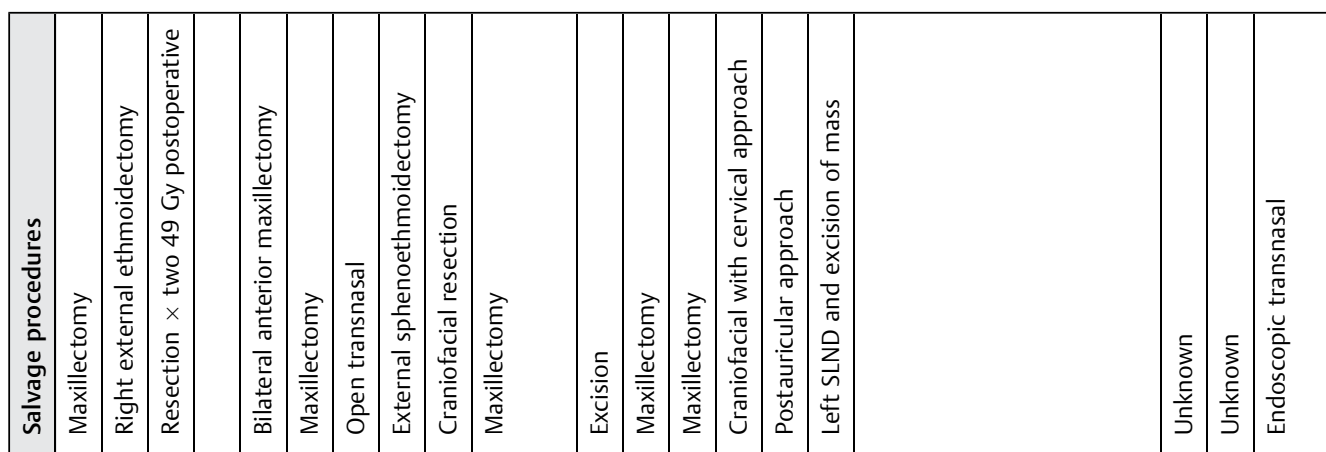

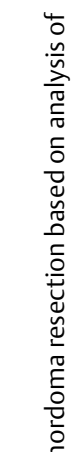

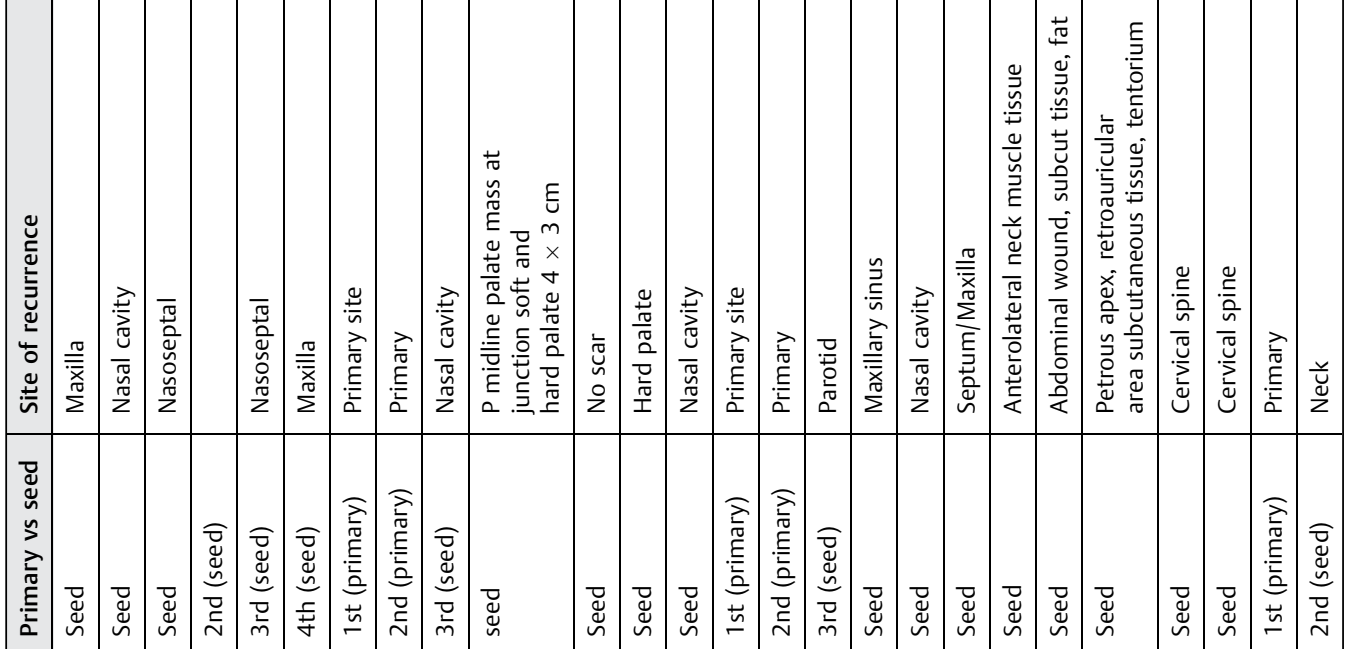

告

蒙

-

促

n

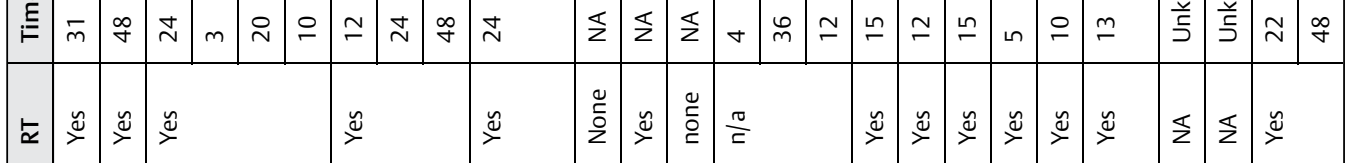

(2)

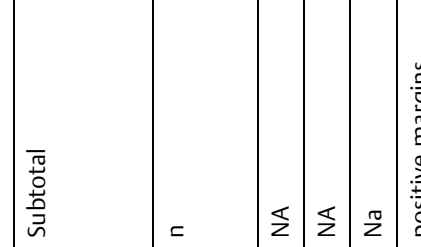

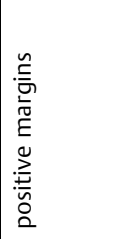

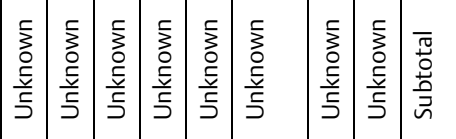

\begin{tabular}{|c|c|c|c|c|c|c|c|c|c|}
\hline 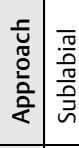 & 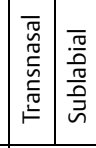 & 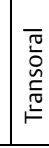 & $\begin{array}{l}\text { 䎡 } \\
\text { 浐 }\end{array}$ & \begin{tabular}{l|l|l|l}
$z$ & $\Sigma$ \\
\end{tabular} & 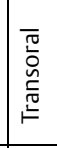 & 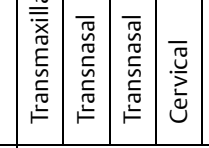 & 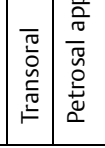 & 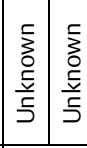 & 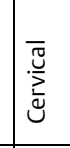 \\
\hline 气゙ & m & & - & m & - & 0 & & $\sim$ & - \\
\hline 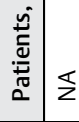 & $\begin{array}{l}尺 \\
11 \\
=\end{array}$ & & $\frac{\mathbb{s}}{z}$ & 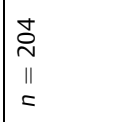 & $\frac{x}{z}$ & $\begin{array}{l}\infty \\
\infty \\
11 \\
\tilde{E}\end{array}$ & & 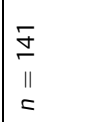 & $\frac{\pi}{z}$ \\
\hline 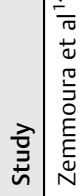 & 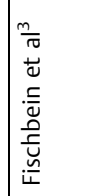 & & 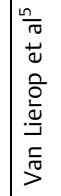 & 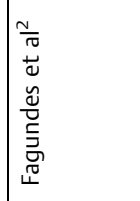 & 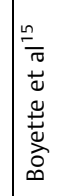 & 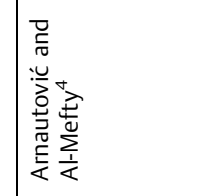 & & 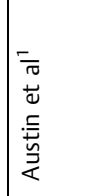 & 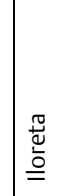 \\
\hline
\end{tabular}

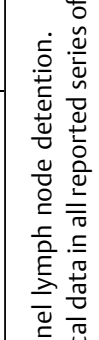

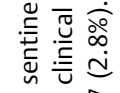

今. 鵖夺

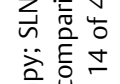

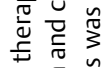

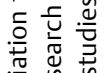

范 
treatment until several years after the transcervical approach, and radiotherapy may have prevented seeding in this case. Lastly, with the advent of advanced endoscopic techniques, utilizing suction instruments such as the Sonopet (Stryker, Kalamazoo, Michigan, United States) or MicroDebrider (Medtronic, Minneapolis, Minnesota, United States) for tumor resection may hypothetically prevent surgical seeding by limiting the exposure of tumor to adjacent sites. However, the argument could also be made that disruption of the tumor with endoscopic instrumentation and piecemeal resection of the tumor could increase the chance of pathway seeding. As the number of chordoma patients treated with an endoscopic endonasal approach continues to grow, we will be better able to compare the incidence of tumor pathway seeding.

Multiple surgical approaches have been used to reach the clivus and can be divided into two main groups: extracranial (transsphenoidal, transoral, transcervical, lateral infratemporal, anterolateral, and posteroinferior) and intracranial (transfrontal, frontotemporal, lateral suboccipital, and medial suboccipital). Each set of approaches has its unique set of advantages and disadvantages. Some of the approaches are associated with significant morbidity and prolonged recovery.

Recent advances in endoscopic sinus surgery and neuroendoscopy have resulted in a new era of minimally invasive skull base surgery. These endoscopic approaches have several distinct advantages compared with traditional approaches. Use of high-resolution optics to provide superior illumination and magnification allows surgeons to have greater confidence in obtaining total or near-total resection of gross tumor. In addition, the use of angled endoscopes allows surgeons to see around structures and into corners to augment tumor resection. Other benefits that have been well documented include the decreased resection of healthy tissue, lack of facial incisions, less postoperative pain, decreased bleeding, and quicker recovery. ${ }^{11-13}$ Endoscopic endonasal techniques facilitate tumor removal with the aid of powered suction instrumentation, in effect offering the potential to minimize the passage of the tumor specimen through the surgical cavity.

Adjuvant therapy is used to augment local control in many of these patients. Our review indicates that adjuvant radiation does not always target therapy along all surgical access routes. We believe adjuvant treatment planning should include consideration of the surgical access route and account for potential risk of tumor seeding on an individual basis. Close surveillance, including imaging of the surgical pathway for potential tumor seeding, is critical in this population.

\section{Conclusion}

To our knowledge, there have been no case reports of surgical seeding following primary tumor resection with endoscopic endonasal techniques. In our patient, tumor resection was performed at different times via both an open transcervical as well as endoscopic endonasal approach, serving as a de facto case control. Tumor recurrence in this instance occurred at the site of previous open transcervical approach, whereas no tumor seeding was noted along the endonasal or transoral pathways. The endoscopic endonasal approach offers the advantage of a more direct/shorter surgical corridor, magnified visualization, and less violation of soft tissue surfaces where tumor implantation can occur.

\section{References}

1 Austin JP, Urie MM, Cardenosa G, Munzenrider JE. Probable causes of recurrence in patients with chordoma and chondrosarcoma of the base of skull and cervical spine. Int J Radiat Oncol Biol Phys 1993;25(3):439-444

2 Fagundes MA, Hug EB, Liebsch NJ, Daly W, Efird J, Munzenrider JE. Radiation therapy for chordomas of the base of skull and cervical spine: patterns of failure and outcome after relapse. Int J Radiat Oncol Biol Phys 1995;33(3):579-584

3 Fischbein NJ, Kaplan MJ, Holliday RA, Dillon WP. Recurrence of clival chordoma along the surgical pathway. AJNR Am J Neuroradiol 2000;21(3):578-583

4 Arnautović KI, Al-Mefty O. Surgical seeding of chordomas. J Neurosurg 2001;95(5):798-803

5 van Lierop AC, Fagan JJ, Taylor KL. Recurrent chordoma of the palate occurring in the surgical pathway: a case report. Auris Nasus Larynx 2008;35(3):447-450

6 Chambers PW, Schwinn CP. Chordoma. A clinicopathologic study of metastasis. Am J Clin Pathol 1979;72(5):765-776

7 Sen C, Triana AI, Berglind N, Godbold J, Shrivastava RK. Clival chordomas: clinical management, results, and complications in 71 patients. J Neurosurg 2010;113(5):1059-1071

8 Camara O, Kavallaris A, Nöschel H, Rengsberger M, Jörke C, Pachmann K. Seeding of epithelial cells into circulation during surgery for breast cancer: the fate of malignant and benign mobilized cells. World J Surg Oncol 2006;4:67

9 Ploumidis A, Panoskaltsis T, Gavresea T, Yiannou P, Yiannakou N, Pavlakis K. Tumor seeding incidentally found two years after robotic-assisted radical nephrectomy for papillary renal cell carcinoma. A case report and review of the literature. Int J Surg Case Rep 2013;4(6):561-564

10 Rolle A, Günzel R, Pachmann U, Willen B, Höffken K, Pachmann K. Increase in number of circulating disseminated epithelial cells after surgery for non-small cell lung cancer monitored by MAINTRAC(R) is a predictor for relapse: a preliminary report. World J Surg Oncol 2005;3(1):18

11 Castelnuovo PG, Delù G, Sberze F, et al. Esthesioneuroblastoma: endonasal endoscopic treatment. Skull Base 2006;16(1): 25-30

12 de Almeida JR, Witterick IJ, Gullane PJ, et al. Physical morbidity by surgical approach and tumor location in skull base surgery. Head Neck 2013;35(4):493-499

13 Lombardi D, Tomenzoli D, Buttà L, et al. Limitations and complications of endoscopic surgery for treatment for sinonasal inverted papilloma: a reassessment after 212 cases. Head Neck 2011;33(8): 1154-1161

14 Zemmoura I, Ben Ismail M, Travers N, Jan M, François P. Maxillary surgical seeding of a clival chordoma. Br J Neurosurg 2012;26(1): 102-103

15 Boyette JR, Seibert JW, Fan CY, Stack BC Jr. The etiology of recurrent chordoma presenting as a neck mass: metastasis vs. surgical pathway seeding. Ear Nose Throat J 2008;87(2):106-109 